\title{
Anormalidades y patologías odontológicas en los equinos
}

\section{Dental abnormalities and diseases in horses}

\author{
Gutiérrez Martínez Daniel Mauricio ${ }^{1}$ e Iregui Iregui Alberto ${ }^{2}$ \\ ${ }^{1} \mathrm{MVZ}$, UNILLANOS y ${ }^{2} \mathrm{MVZ}$, Esp (c)MSc. Docente Programa de Licenciatura en \\ Producción Agropecuaria UNILLANOS \\ airegui@unillanos.edu.co
}

Recibido 11 de Diciembre 2015, Aceptado 26 de Mayo 2016

\section{RESUMEN}

Los problemas dentales en los equinos son de gran importancia en la práctica veterinaria, puesto que, sí no son tomados en cuenta se puede estar incurriendo en dificultades mayores que provocan grandes daños digestivos en el animal, comprometiendo su salud. Este trabajo se enfocó en estudiar y analizar los problemas odontológicos que presentan los equinos estabulados en fincas de Villavicencio, Meta, Colombia. Inicialmente se describieron las anormalidades y patologías dentales, y una vez diagnosticados se instauraron los tratamientos de acuerdo al problema encontrado, además se realizó la estimación de la prevalencia de casos clínicos y subclínicos. Se encontró que el $100 \%$ de los equinos evaluados presentaron alguna patología odontológica, y apenas el 53\% de los propietarios accedió a realizar alguna parte del tratamiento recomendado, se destaca que el resultado de los tratamientos, mejoraron el peso del animal en $9 \%$ en un periodo de dos meses, así como su manejo con la rienda; el sarro y cálculo dental son la segunda patología más frecuentes en equinos después de las espículas dentales u odontofitos. Además se observó en los equinos una relación braquignatismo, problemas comportamentales, y de rienda, sin importar cuál sea la actividad que se realice con el equino.

Palabras clave: Odontofitos, cálculo dental, prognatismo, braquignatismo, haba. 


\begin{abstract}
Dental problems in horses are of great importance in veterinary practice, because if they are taken into account may be incurring higher digestive difficulties caused great damage to the animal, compromising their health. This work was focused on studying and analyzing the dental problems presented in horses stabled in farms of Villavicencio city, Meta, Colombia. Initially abnormalities and dental pathologies were described, and once diagnosed the treatments were initiated according to the problem found, moreover was performed to estimate the prevalence of clinical and subclinical cases. It was found that $100 \%$ of those tested showed some equine dental pathology, and only $53 \%$ of owners agreed to make some part of the recommended treatment, is highlighted that the result of the treatments, improved animal weight $9 \%$ in a period of two months, well as its management with reins; tartar and dental calculus are the second most common dental pathology in horses after spicules or odontophytes. It was further noted in horses one brachygnathism, behavioral problems, and rein relationship, no matter what the activity done with the horse.
\end{abstract}

Keywords: Odontophytes, dental calculus, prognathism, brachygnathism, bean.

\title{
RESUMO
}

Os problemas dentários em cavalos são de grande importância na prática veterinária, porque sim não são tomados em consideração se pode estar causando maiores dificuldades digestivas que causou grande dano ao animal, comprometendo a sua saúde. Este trabalho se concentrou em estudar e analisar os problemas dentários que ocorrem em cavalos estabulados em fazendas no Villavicencio, Meta, Colombia. Inicialmente anormalidades e patologias odontológicas foram descritos, e os tratamentos foram iniciados uma vez diagnosticado de acordo com o problema encontrado, além foi estimado da prevalência de casos clínicos e subclínicos. Verificou-se que $100 \%$ dos equinos que foram testados mostraram alguma patologia dentária, e apenas 53\% dos proprietários concordaram em fazer alguma parte do tratamento recomendado, é 
salienta que o resultado dos tratamentos, melhoradas peso do animal $9 \%$ num período de dois meses, e manipulação de as rédeas; tártaro e cálculo dentário são o segundo patologia dental mais comum em cavalos após espículas ou odontofitos. Foi ainda observado em cavalos, uma relação braquygnatismo, problemas comportamentais, e rédea, não importa o que a atividade feita com o cavalo.

Palavras-chave: Odontófitos, cálculo dentário, prognatismo, braquygnatismo, feijão.

\section{INTRODUCCIÓN}

Los problemas dentales en los equinos son de gran importancia en la práctica veterinaria, que en la mayoría de casos no son tomados en cuenta, lo cual puede conllevar a dificultades mayores que provocan grandes daños en el animal; estos problemas se pueden relacionar con la aprehensión de alimentos, mala masticación y deglución, y también con el mal uso de los frenos en el trabajo con los caballos. Es importante resaltar que la aparición de alteraciones dentales afecta de diversas formas la salud y compromete la digestión del animal, por lo tanto este trabajo fue realizado con un enfoque en los problemas odontológicos que se presentan en equinos estabulados en fincas del municipio de Villavicencio, Meta.

\section{Examen clínico de la boca del equino}

Los tejidos blandos se comienzan a evaluar desde que se abre la boca del equino para determinar el color de las mucosas, las cuales en animales sanos se observan brillantes y cubiertas por una película delgada de saliva (Figura 1); esto no necesariamente se tiene que hacer metódicamente sino de manera práctica para no incomodar al equino por tener mucho tiempo la boca abierta sumado a la manipulación. Los factores a tener en cuenta al realizar este examen es la coloración e integridad de las mucosas, y que no evidencie signos de inflamación ni de crecimientos neoplásicos en los labios, encías y espacios interdentales, paladar, carrillos y lengua, valorando además su funcionalidad. 
Periodonto: Se debe tener claro que el periodonto puede estar inflamado normalmente cuando están mudando los dientes deciduos por los permanentes, esto a la edad de 2.5 a 4.5 años. El periodonto se observa a simple vista y se determina si tiene signos de inflamación (gingivitis), o alguna neoplasia, o herida, o si por el contrario se encuentra normal, para que la evaluación dental sea más relevante, se recomienda realizarla en dos fases las primera, la primera consiste en examinar la integridad, morfología y funcionalidad de los incisivos y los colmillos, en los machos esto se realiza levantando el labio superior para observar los incisivos (101 a 104, 201 a 204, 301 a 304 y 401 a 404) examinando también los premolares, principalmente los superiores pasando una mano entre el carrillo y estos para detectar las espículas dentales u odontifitos, que es la patología dental más común en los equinos estabulados; en la segunda fase se utiliza el abre bocas o especulo oral equino (Figura 2), para poder observar con ayuda de una linterna las anormalidades y patologías que se presentan en los carrillos, premolares y molares del equino, el examen va acompañado de una palpación de estas estructuras, teniendo en cuenta que estén todos los dientes, el rasamiento normal, y la integridad y coloración de los mismos, puesto que en el carrillo se pueden ver heridas o laceraciones causadas por las espículas dentales y neoplasias.

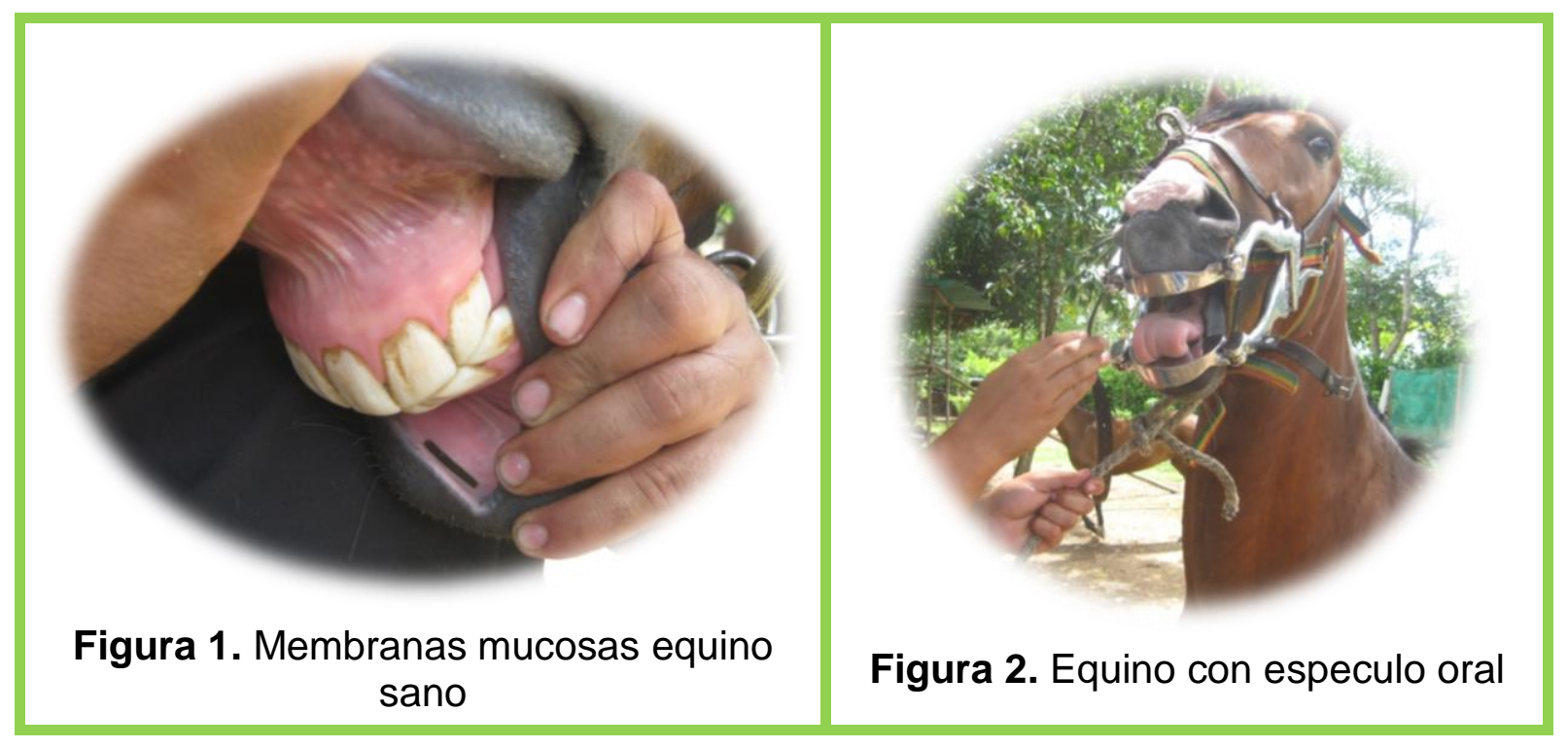


Para realizar mejor el examen odontológico en equinos puede ser necesario la tranquilización del paciente, para esto se utiliza acepromazina o xilacina, y dependiendo de las circunstancias puede ir acompañada de un método de restricción como el axial, o se puede usar solo el método de restricción dependiendo el manejo que haya tenido el equino o la nobleza de este.

\section{Principales anormalidades y patologías odontológicas en los equinos}

Los problemas de oclusión son frecuentes, y de dos tipos: 1) Problemas en la formación de la mandíbula, y 2) Problemas únicamente de los incisivos; dentro de los primeros hay dos: braquignatismo (boca de loro), el cual consiste en que la mandíbula inferior es más corta, y el inverso es el prognatismo (boca de mono), mandíbula inferior es más larga (Cruz, 2004). Por otro lado, la mal-oclusión por problemas de los incisivos se dividen en cuatro: 1) boca de sonrisa, como su nombre lo indica los incisivos del equino se observan como si se estuviera riendo, 2) inclinada o diagonal, en ésta los incisivos rasan más de uno de los dos lados y se observan como si cayeran en diagonal, 3) escalera o irregular, su nombre indica que el rasamiento de los incisivos es irregular y estos se observan como si fuera una escalera unos más altos que los otros, y 4) el rasamiento excesivo ocasiona cara de enojo o triste.

\section{Anormalidades odontológicas:}

Son casos que no son normales para los equinos, aunque no sean un problema y no son causadas por trauma, mal manejo o un agente infeccioso como las que se van a describir a continuación:

a) Diente de lobo: Es el primer premolar de cada arcada es decir la pieza número cinco según la nomenclatura Triada, según la cual se denominarán 105, 205, 305 ó 405 dependiendo de su ubicación (Dixon, 2002), aunque generalmente sale en la parte superior, entre los 6 y los 18 meses de edad. Este se nombra como anormalidad porque suele causar varios problemas entre los que están el dolor intenso, ulceras en la mucosa del carrillo y presión sobre el freno (Cardona y Álvarez, 2010). 


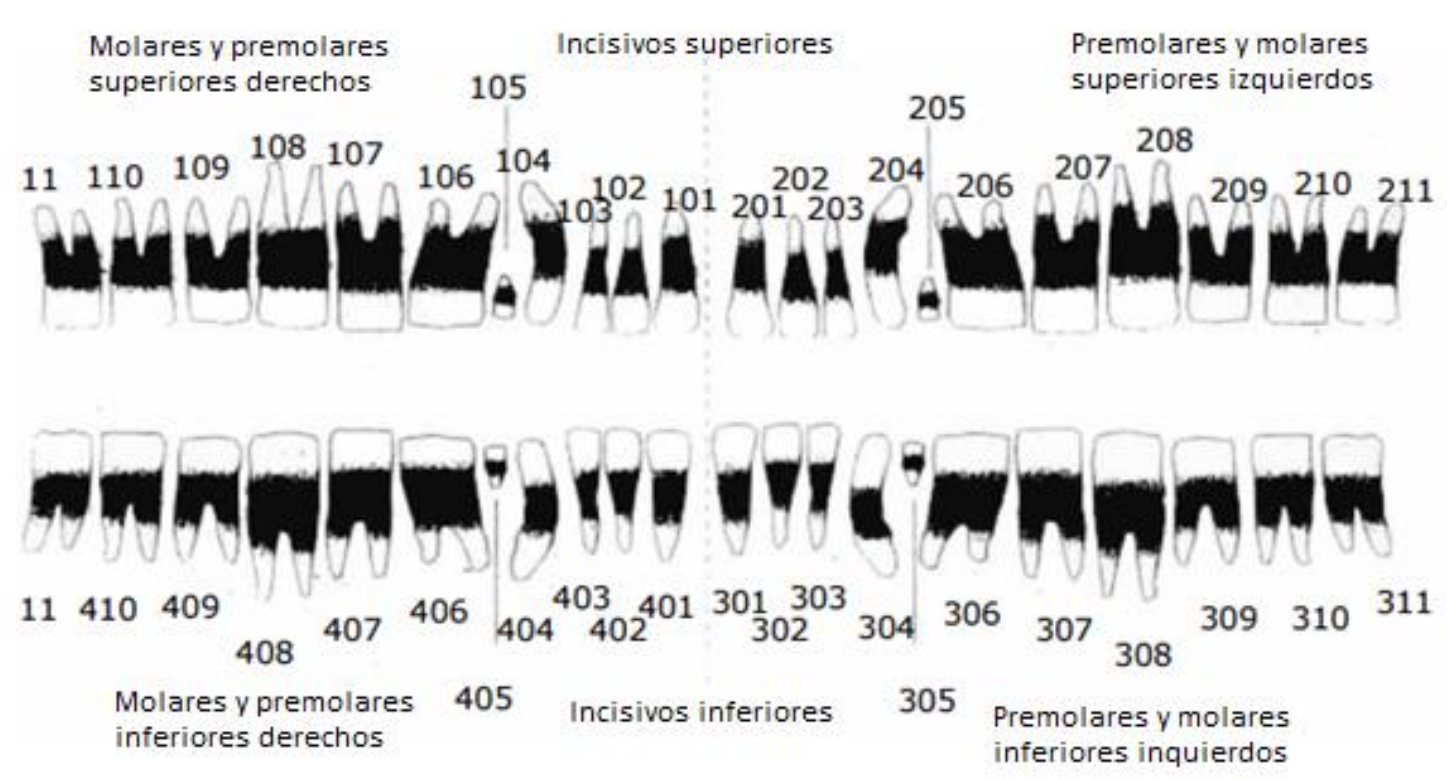

Fuente: Modificado de Dixon (2002).

b) Colmillos en yeguas: Los colmillos son dientes que salen una sola vez en la vida de equinos y generalmente emergen en machos, en yeguas salen de forma rudimentaria y según Dixon y Dacre, (2005) tiene una incidencia del $27.8 \%$, no se ha encontrado una causa directa pero también se ha observado que las yeguas se tornan agresivas y territoriales (Cardona y Álvarez, 2010).

c) Braquignatismo: La mandíbula inferior más corta que la superior conduce a una sobre mordida; en la mayoría de los casos, el defecto se limita a maloclusiones de los incisivos debido al acortamiento del componente rostral de la mandíbula y del espacio interdental.

d) Prognatismo: La situación se invierte al braquignatismo, la mandíbula esta alargada respecto al maxilar, comúnmente se le denomina "belfo".

e) Mal-oclusión: Además del braquignatismo y el prognatismo hay otras cuatro anormalidades que se pueden encontrar en el examen clínico: 1) boca de sonrisa, 2) inclinada o diagonal, 3) escalera o irregular, y 4) cara de enojo, en la cual los incisivos se observan como una cara triste o enojada. 
f) Hipoplasia dental: Esta anormalidad no es muy común pero se observó en un caso el cual tenía hipoplasia de los caninos, pero no tenía ningún problema evidente; este problema es genético y se presenta por un daño en la germinación dental.

g) Oligodoncia: Es la ausencia de uno o varios dientes, la cual suele ser el resultado o la secuela de la enfermedad periodontal o dental; cuando falta un solo diente los dientes vecinos se mueven generando un acortamiento de la arcada, y esto genera una oclusión anormal y cambio en el desgaste de los dientes (Baker y Easley, 2002); cabe resaltar que se debe tener en cuenta que el equino no esté en la edad que muda los dientes deciduos y realizar una radiografía para verificar que se encuentra ausento o si no hubo erupción.

\section{Patologías odontológicas:}

Son aquellas causadas por trauma, manejo, agentes infecciosos o algunas veces se desarrollan por alguna anormalidad, que terminan generando una alteración en la morfología y fisiología odontológica del equino.

a) Odontofitos: Es la patología odontológica más frecuente en los equinos, puesto que se ha estimado que el $100 \%$ de los animales estabulados la presentan debido al consumo de concentrado, porque el equino realiza más movimientos verticales que laterales, lo cual hace que los bordes vestibulares de las piezas superiores y los bordes linguales de las inferiores no se desgasten apropiadamente generando unas puntas (Dixon y Dacre, 2005), las cuales traumatizan la mucosa de los carrillos y la lengua, causando laceraciones y por ende con el tiempo ulceraciones dolorosas, lo cual genera bastante dolor, disminuyendo la masticación y por ende no se realiza una buena digestión del alimento predisponiéndolo a cólicos, bajando su condición corporal.

b) Rampa: Generalmente es consecuencia de la mala oclusión ya sea el braquignatismo 0 el prognatismo, generando un desgaste anormal principalmente en los dientes 106, 206, 306 y 406, dependiendo de la 
severidad estas rampas que se ubican rostralmente a la mandíbula, trasmiten una presión extrema a las articulaciones temporo-mandibulares, y debido a las fuerzas mecánicas provocadas a los segundos premolares inferiores, se puede llegar a crear un diastema, en el cual se queda atrapada la comida, generando con el tiempo una enfermedad periodontal (Johnson y Porter, 2006).

c) Desgaste anormal en premolares y molares: Además de las rampas que se presentan hay tres formas de desgaste anormal que son las más comunes:

1. Desgaste transversal exagerado $\mathbf{u}$ ondulaciones: Este excesivo desgaste se presenta principalmente en los premolares y los molares formándose ondulaciones en su superficie oclusal; en cada diente existen de dos a tres o más de estas ondulaciones, que son creadas por plegamientos del esmalte dental, $y$ en algunos animales con mordida anormal, aumentando estos surcos de manera exagerada en la mandíbula y el maxilar, de tal forma que cuando el animal cierra la boca, las arcadas dentales se traban, impidiendo los movimientos rostro-caudales de la mandíbula, y específicamente de la articulación temporo-mandibular (Dixon y Dacre, 2005); el tratamiento consiste en el limado de estos pliegues.

2. Desgaste excesivo: El desgaste excesivo de la cara oclusal de los premolares y molares se da por causas diversas, como vejez y anormalidades del desgaste en los incisivos (boca en sonrisa); los caballos presentan un desgaste normal de 2-3 $\mathrm{mm}$ por año de los premolares y molares; si bien la reserva de crecimiento reemplaza la superficie gastada, en los animales con edades avanzadas ( 25 a 30 años) esta reserva se agota y los dientes se desgastan excesivamente (Dixon, 2003). El desgaste excesivo también puede ser causado por la poca dureza de los tejidos dentales, en estos dientes la superficie solo consta de dentina y cemento ya que se ha desgastado el esmalte; los alimentos son comprimidos pero no triturados provocando gastritis y enteritis con pérdida de condición corporal (Cruz et al., 2010). 
3. Boca onda: Se presenta cuando la superficie oclusal de varios dientes se desgasta en forma irregular y adquieren forma de onda; esté defecto es común y con el tiempo empeora si no se corrige, el problema puede observarse en un solo lado o en ambos lados de la boca; su etiología es desconocida, pero se cree que tiene que ver con la diferencia de erupción de los dientes permanentes.

d) Sarro dental: Es la acumulación de sales de calcio y fósforo sobre la superficie dental.

e) Calculo: El sarro no es una patología relevante en los equinos pero el problema es cuando se acumula generando el cálculo dental que puede desarrollar una gingivitis, pero rara vez llevan a la enfermedad periodontal. Los casos severos presentan resorción del hueso y daño del aparato periodontal, siendo más común la enfermedad en los animales viejos (Easley, 2004). Los cálculos suelen afectar los dientes caninos, y se presenta generalmente en la arcada inferior, e inician de un tamaño mínimo y pueden aumentar progresivamente, y cuando están grandes causan úlceras a nivel de los labios; sin embargo, estos cálculo se pueden retirar fácilmente con fórceps (Dixon, 2003).

f) Enfermedad periodontal: La enfermedad periodontal afecta a los equinos de todas las edades, sin embargo, hay mayor incidencia en los caballos viejos, siendo causa de dolor y la pérdida prematura de los dientes (Dixon y Dacre, 2005). La flora bacteriana normal de los equinos son bacterias Gram positivas, pero cuando aumentan las bacterias Gram negativas aeróbicas, anaerobias y espiroquetas dan como resultado procesos inflamatorios con posterior degradación y pérdida de los tejidos más profundos del ligamento periodontal (Klugh, 2005). La enfermedad también puede ser secundaria a los diastemas, a los desplazamientos hacia medial o lateral de los premolares y molares, a los crecimientos dentales excesivos, a las mal-oclusiones, a las anormalidades como boca en onda, por lo tanto la corrección del problema primario puede 
ayudar en la resolución de la enfermedad periodontal, a menos que se encuentre muy avanzada (Dixon y Dacre, 2005); sus estadios son:

1. Etapa cero, periodonto normal: Se caracteriza por la encía rosada, que se adhiere firmemente a los dientes y se ajusta estrechamente a la arcada dental, la encía se encuentra húmeda, la superficie es lisa y la profundidad del surco gingival es de $5 \mathrm{~mm}$ o menos, es decir una encía sana, la cual es mencionada para tener en cuenta los cambios que se van presentando

2. Etapa uno, gingivitis: Se caracteriza por la encía inflamada y rojiza, el surco gingival tiene una profundidad normal, pero puede sangrar en el sondeo.

3. Etapa dos, inicio de la enfermedad periodontal: Se reconoce por la presencia de forraje, desechos de la alimentación retenidos en los espacios que se forman. Esta etapa se caracteriza por la pérdida hasta de un $25 \%$ del periodonto, la profundidad de sondaje es superior a $5 \mathrm{~mm}$, la encía se encuentra retraída, ulcerada, el cemento supragingival se degrada, existe pérdida de hueso alrededor de los dientes y la movilidad dental se presenta de forma ligera.

4. Etapa tres, moderada enfermedad periodontal: En esta etapa se encuentran espacios o bolsillos en las piezas dentarias, lesiones de la encía y el epitelio circundante son similares a la etapa anterior, pero aumenta la profundidad de sondaje y a veces supera la longitud de la sonda; esta etapa se caracteriza por la pérdida de un 25 a 50\% del periodonto y la pérdida ósea es menor al $50 \%$ en torno a la raíz del diente.

5. Etapa cuatro, grave o avanzada enfermedad periodontal: En este caso se observa en el examen clínico retracción gingival severa, ulceración, edema, degradación del cemento supragingival y subgingival, necrosis del epitelio circundante, pudiéndose presentar descarga purulenta, la movilidad dental se encuentra aumentada; esta etapa se caracteriza por la pérdida de un $50 \%$ del periodonto, además se observan cambios radiográficos como 
pérdida de hueso alveolar en un $50 \%$, desgaste de los ápices, y cambios en la corona y las raíces.

g) Diastema: Es el espacio entre dos dientes. El diastema en los premolares y molares es una de las principales causas de enfermedad periodontal en equinos pero no es muy fácil de identificar aparte de que no es muy común (Rucker, 2006), se debe recordar que en los equinos, todos los dientes de una arcada están muy próximos entre sí, de tal manera que no quedan espacios interdentales que permitan el paso y la acumulación de los alimentos; mientras que el diastema en los incisivos no causa importantes trastornos de aprehensión, si bien, el alimento acumulado genera inflamaciones y retracción gingival, se debe limpiar con un cepillos dos veces por semana para evitar estos problemas. No sobra aclarar que los diastemas más pequeños (de $1 \mathrm{~mm}$ ) se pueden desarrollar en la superficie oclusal en algunos caballos viejos, y generalmente no causan problemas clínicos porque no tienden a atrapar y compactar la comida, cuando los diastemas son más anchos ( $>5 \mathrm{~mm}$ ) pueden atrapar comida causando periodontitis y retractación gingival (Collins y Dixon, 2005).

h) Fractura de incisivos: Son ocasionadas generalmente por traumas severos, si no se tratan a tiempo pueden generar caries por problemas infecciosos. Cuando hay una fractura o pérdida de un incisivo el diente opuesto tiende a crecer exageradamente y debe ser limado dos veces al año, de no hacerlo así se desarrollan los Ilamados "escalones" (Crabill y Schumacher, 1998).

i) Incisivo astillados: Estas son fracturas menores de la superficie oclusal de los dientes incisivos, y se producen debido al comportamiento anormal o los llamados comúnmente "vicios" que tienen algunos caballos, los cuales desgastan de forma anormal los incisivos cuando muerden objetos duros como madera, cemento o metal, lo cual es generado por el estrés de los caballos que se encuentran estabulados. 
j) Caries: Es una enfermedad de los tejidos calcificados del diente, resultado de la acción de los microorganismos sobre los hidratos de carbono de la cavidad oral, y se caracteriza por la desmineralización de la parte inorgánica y destrucción subsiguiente de la parte orgánica del diente (Dacre, 2006). Las caries del infundíbulo en los incisivos son raras, pero en los premolares y molares existen en algún grado en la mayoría de caballos de avanzada edad que son examinados; la afección es más común en el infundíbulo debido al relleno incompleto de esta estructura del diente con cemento. Todos los defectos del cemento dentro del infundíbulo, permiten que se acumule en el centro del diente la comida y las bacterias, dándose la fermentación y producción de ácido, lo cual lleva a descalcificación del cemento circundante y con el tiempo se afecta el esmalte y la dentina, pudiéndose provocar abscesos apicales causando sinusitis y tractos fistulosos. Los síntomas de las caries dentales son cambios de la coloración normal del diente, generalmente a color amarillo pardo o pardo negruzco, aspereza y pérdida de sustancia con formación de fosas y cavidades, a veces ocurre fractura espontánea del diente, olor fétido y odontalgia cuando queda al descubierto la pulpa, o pulpitis purulenta, lo cual coincide con trastornos en la masticación caracterizados por una masticación lenta, interrumpida, con salivación y presencia de acumulación de alimento, además la caries puede llegar a producir fistulas dentales (Cruz et al., 2010).

k) Haba o hiperplasia del paladar duro: Esta se genera inmediatamente por detrás del arco formado por los incisivos superiores, originando mucho dolor durante la aprehensión y masticación de los alimentos, es un problema frecuente que genera inapetencia y pérdida de peso progresiva del animal. No todas las palatitis son patológicas; existe una fisiológica que puede presentarse durante la erupción de los incisivos permanentes y la caída correspondiente de los deciduos, alrededor de los dos años y medio (Cruz, 2004), se genera por consumir alimentos muy fibrosos o forrajes muy cortos. 
I) Heridas: Son injurias que se presenta en la piel o la mucosa por algún trauma en el paladar, encías, labios, lengua y carrillos, pero los más comunes son las últimas tres.

\section{METODOLOGÍA}

Este trabajo se realizó en nueve fincas de Villavicencio, Colombia, realizando exámenes dentales a 32 equinos, 19 machos y 13 hembras con el fin de detectar problemas odontológicos como normalidades y patologías, y de esta manera aplicar los tratamiento adecuados, además se estimó la prevalencia de estos problemas dentales.

\section{RESULTADOS Y DISCUSIÓN}

\section{Tratamientos realizados}

Las anormalidades y patologías que se nombraron anteriormente fueron las que se encontraron en los equinos que se examinaron en nueve fincas diferentes, es por eso que a continuación se muestra los tratamientos que se propusieron en los pacientes y al final se muestra un análisis estadístico de las patologías y anormalidades odontológicas que se presentaron en los equinos.

\section{Profilaxis dental}

Comprende varios procedimientos tales como el limado (Figura 3), el cual se realiza con: 1) lima recta que se mueve de forma craneal a caudal para los premolares y molares inferiores y 2) lima angulada que se hace de forma lateral para los premolares y por la parte medial en los molares superiores, esto con el fin de eliminar y redondear las puntas de esmalte de los premolares y molares (odontofitos), y además con estas limas se pueden eliminar y nivelar las rampas en los primeros premolares dependiendo del caso (106, 206, 306 y 406) (Figura 4) para así eliminar las ondulaciones; es decir que con este procedimiento se corrigen tres patologías, por lo tanto es necesario que se realicé por lo menos dos veces al año, aunque es recomendable por experiencia que el limado se efectué tres veces al año porque el equino se siente mejor. 


\section{Nivelación de incisivos}

Esta nivelación se realiza con discos de diamantes o con cualquier tipo de fresa que se maneja dentro de la profilaxis dental, es relativamente sencillo para nivelar el desgaste y la oclusión de incisivos con este procedimiento se pueden corregir los incisivos astillados, la oclusión en boca de sonrisa, en enojo y los desgastes anormales que se pueden ir generando por el prognatismo y braquignatismo. En los equinos estabulados este procedimiento se debe realizar una vez al año, puesto que estos ejemplares no usan sus incisivos.

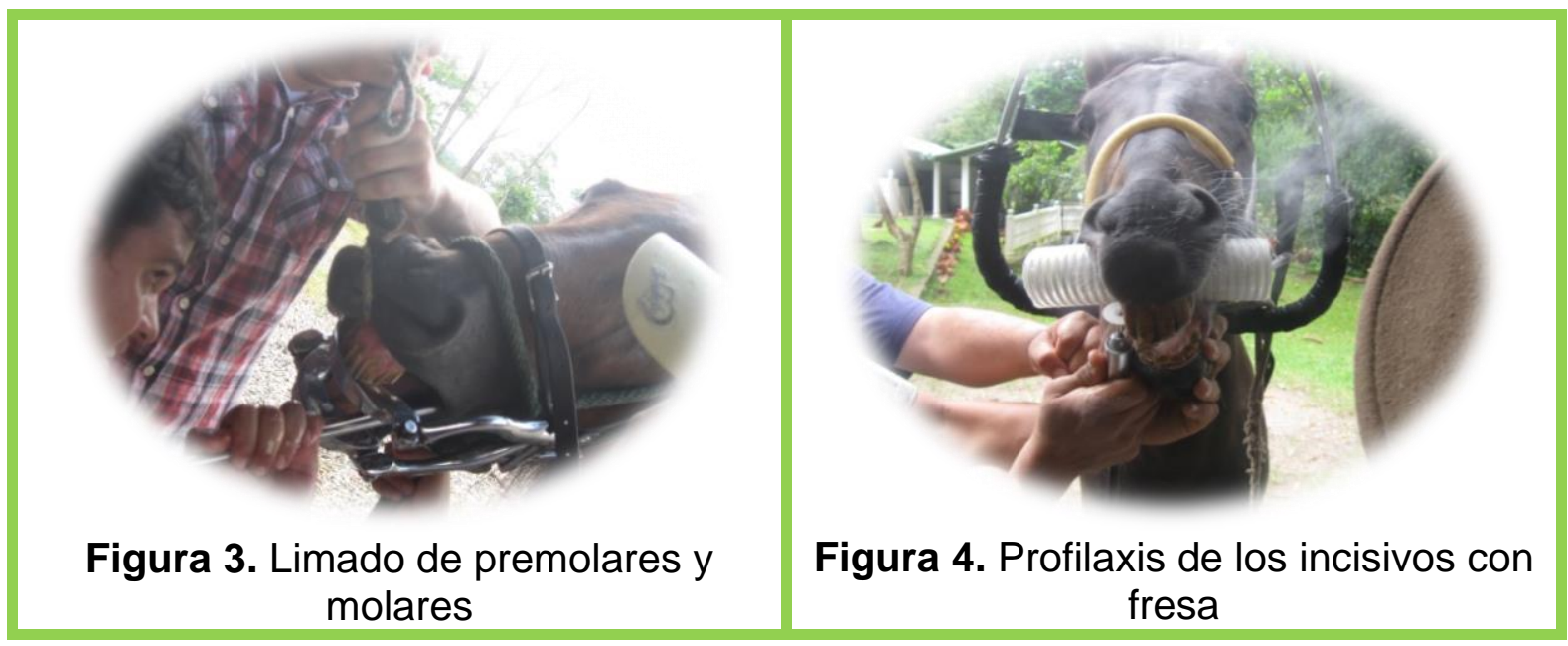

\section{Tratamiento para dientes fracturados}

Las fracturas dentales se pueden restaurar con resinas de fotocurado, o también con amalgama dental que son los mismos procedimientos que se realizan en humanos con diferencia en la cantidad usada, pero no en todos los casos es primordial realizar estos tipos de tratamientos porque depende del diente que este afectado; además es necesario realizar limpieza para evitar que las caries avancen.

\section{Eliminación de sarro y calculo dental}

Se hace principalmente con una cureta dental, para raspar los incisivos y los colmillos, eliminando ese cálculo dental, lo cual no es una patología crítica puede 
derivar en enfermedad periodontal, que si es grave, por lo tanto es preferible prevenir haciendo control temprano.

\section{Corrección de prognatismo y braquignatismo}

No se realiza con frecuencia, es un procedimiento complejo que consiste en recortar los cóndilos, y debe realizarla un médico veterinario especializado en cirugía, siendo necesario en equinos de paso cuando es muy marcado el prognatismo y braquignatismo causando descalificación en exposiciones.

\section{Extracción del diente de lobo}

Se debe resaltar que cualquier procedimiento de extracción de un diente debería ir acompañada de una radiografía, pero en nuestro medio, es muy difícil cumplir todas las expectativas, y en estos dientes no es tan necesario, ya que su raíz es muy corta (Figura 6). Para realizar la extracción del diente de lobo se debe tranquilizar el equino con Acepromacina a una dosis de $0.08 \mathrm{mg} / \mathrm{kg}$ de peso, luego se comienza a desbridar la encía hasta aflojar el diente, posteriormente con una pinza de extracción se saca sujetándolo y moviéndolo para los lados y hacia afuera (Figura 5), luego se limpia con agua sal.

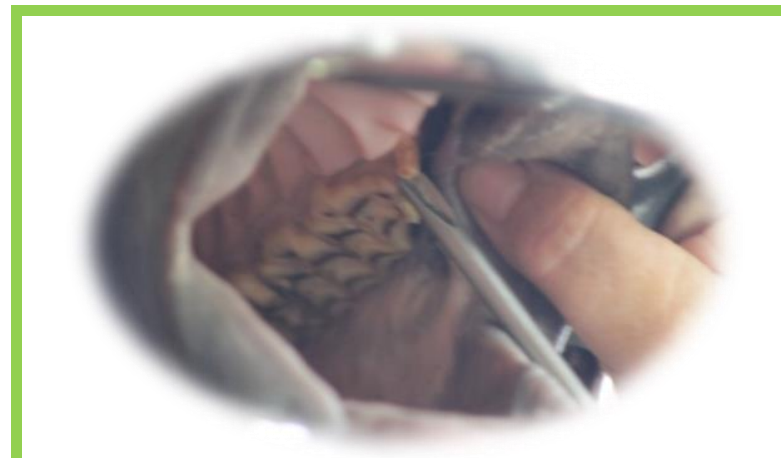

Figura 5. Remoción de diente de lobo

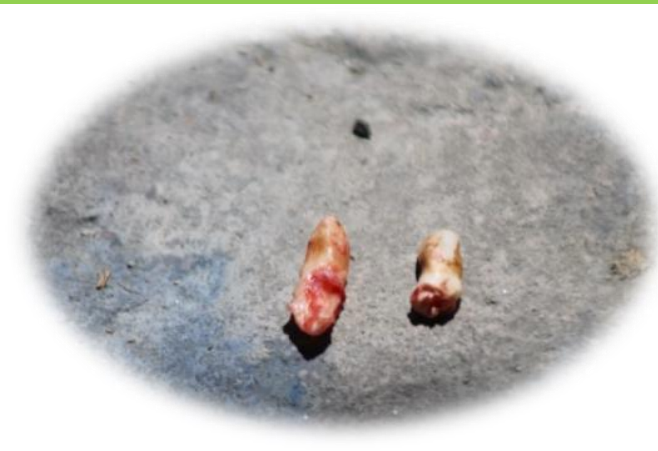

Figura 6. Dientes de lobo extraídos

\section{Recesión de la hiperplasia del paladar superior (haba)}

Este procedimiento quirúrgico es un poco rustico, luego de realizar el protocolo de tranquilización elegido, se procede a derribar el paciente por el método que más convenga, una forma es restringir las cuatro patas, luego se usa un cuchillo 
previamente desinfectado, en lugar de un bisturí porque es un tejido muy fuerte y la hoja de bisturí se puede romper y el caballo fácilmente la puede ingerir, lo cual es peligroso ocasionando un problema grave, con este cuchillo y una pinza anatómica se procede a retirar el exceso de tejido (Figuras 7 y 8), finalmente se realiza un baño con una solución de agua con sal durante 3 días.

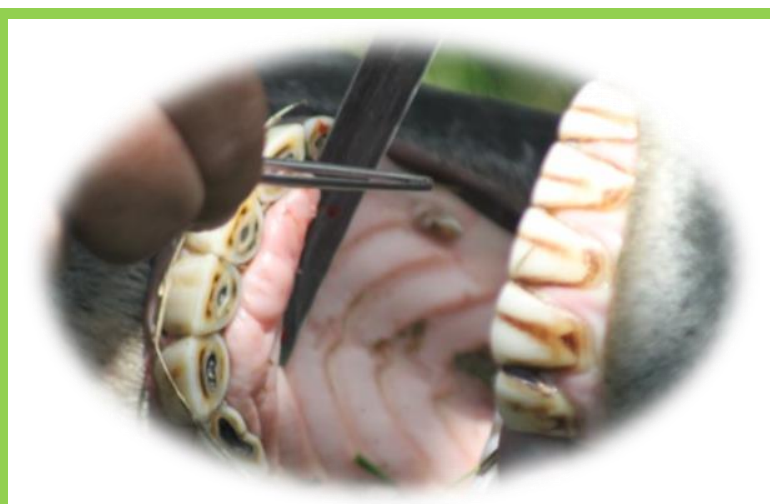

Figura 7. Recesión de Haba

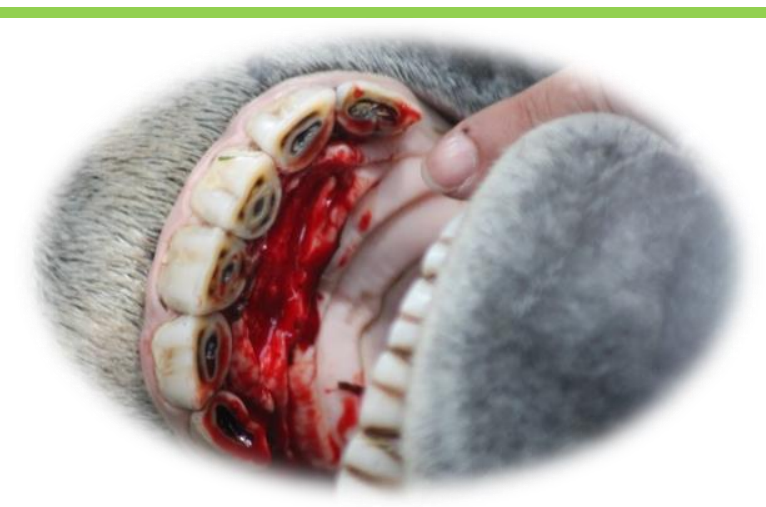

Figura 8. Luego de la recesión del haba

\section{Extracción de los incisivos retenidos}

Es un procedimiento sencillo (Figuras 9 y 10), porque el incisivo está casi suelto, pero no se cae, por lo tanto, es necesario extraerlo porque se acumula comida dentro de éste, y se convierte en un foco de infección, el procedimiento se trata de sujetar el diente con una pinza de extracción y hacerle palanca para extraerlo.

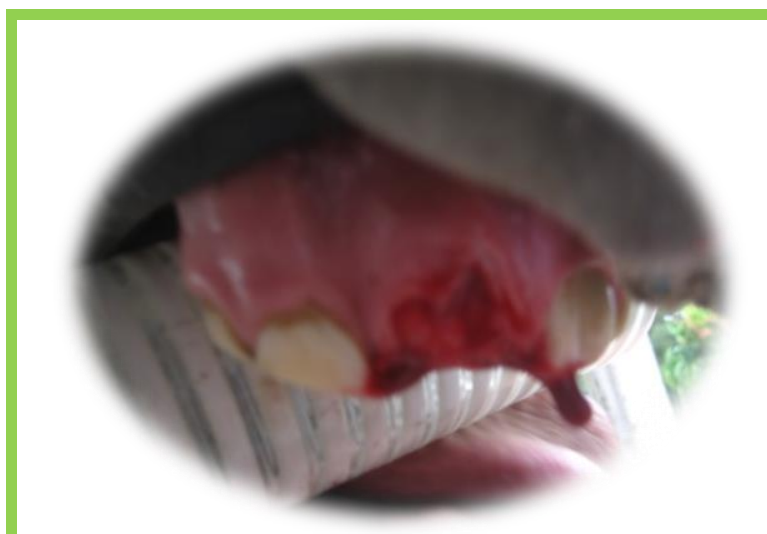

Figura 9. Extracción de incisivo

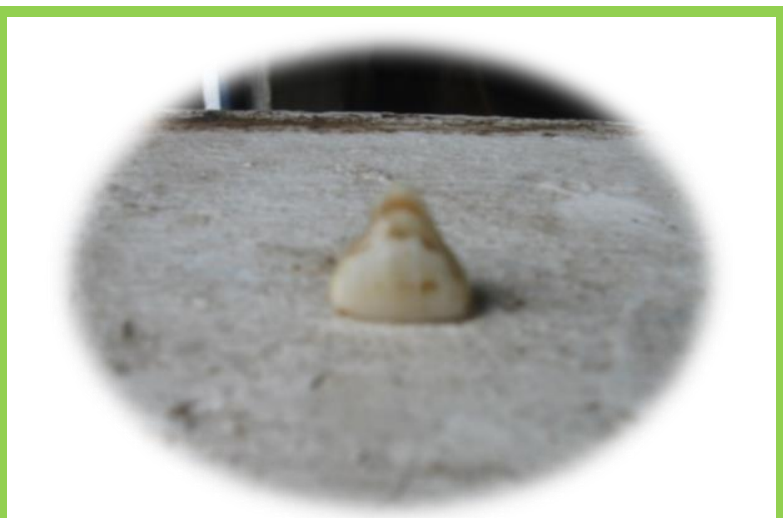

Figura 10. Incisivo que tenía retenido 
Se examinaron 32 equinos de los cuales 19 fueron machos y 13 hembras. Se resalta que el $100 \%$ de los equinos evaluados presentaban al menos dos patologías odontológicas, cabe destacar que ningún equino presentó solamente anormalidades, como se sabe, estas se convierten en el futuro en patologías odontológicas. Los equino con solo patologías fueron 14 que corresponde al $43.75 \%$ y 18 animales (56.24\%) presentaron anormalidades y patologías.

Las yeguas son más predispuestas a tener anormalidades, a pesar de que el numero hembras con respecto a los machos en la población estudiada fue inferior en $9 \%$, para determinar su prevalencia, se realizó un análisis por separado de cada anormalidad y patología (Tabla1). En las anormalidades se observó que no se presentaron de forma independiente, generalmente van acompañadas de alguna otra patología, y que la yeguas son las más afectadas con más de una anormalidad con relación a los machos (Tabla 1).

Tabla 1. Anormalidades y patologías por genero

\begin{tabular}{|c|c|c|c|c|}
\hline \multirow[b]{2}{*}{ Grupo } & \multicolumn{2}{|c|}{ Yeguas } & \multicolumn{2}{|c|}{ Caballos } \\
\hline & Número & $\begin{array}{c}\text { Porcentaj } \\
\text { e (\%) }\end{array}$ & Número & $\begin{array}{c}\text { Porcentaj } \\
\text { e }(\%)\end{array}$ \\
\hline $\begin{array}{c}\text { Equinos que presentaron } \\
\text { únicamente patologías }\end{array}$ & 3 & 21,4 & 11 & 78,5 \\
\hline $\begin{array}{l}\text { Equinos que presentaron } \\
\text { anormalidades y patologías }\end{array}$ & 10 & 55.5 & 8 & 45,5 \\
\hline Una anormalidad & 6 & $46,2^{1}$ & 7 & $36,8^{2}$ \\
\hline Dos anormalidades & 4 & $30,8^{1}$ & 1 & $5,3^{2}$ \\
\hline
\end{tabular}

Considerando población de: ${ }^{1}$ solo yeguas y ${ }^{2}$ Solo caballos

En los exámenes se detectaron 23 anormalidades en toda la población equina evaluada, cinco equinos presentaron dos patologías, los equinos que presentaron mala oclusión tenían diente de lobo, y en el caso de las yeguas y caballo que tenían dos anormalidades presentaron colmillos hipoplásicos (Tabla 2).

La anormalidad más frecuente fue la presencia de diente de lobo, particularmente una yegua presento los cuatro dientes, (dos superiores y dos inferiores) lo cual es muy raro. También, de los exámenes se puede deducir que las yeguas fueron las 
únicas que presentaron braquignatismo, por lo tanto, en la población estudiada tienen mayor predisposición a sufrir esta anormalidad, lo cual tiene un mayor porcentaje de incidencia (15.6\%) con respecto a las otras anormalidades.

Tabla 2. Anormalidades en el total población equina observada

\begin{tabular}{cccc}
\hline Anormalidades & $\begin{array}{c}\text { Número } \\
\text { Yeguas }\end{array}$ & $\begin{array}{c}\text { Número } \\
\text { Caballos }\end{array}$ & $\begin{array}{c}\text { Total } \\
\text { Población (\%) }\end{array}$ \\
\hline Colmillos en yeguas & 3 & 0,0 & 9,37 \\
Dientes de lobo & 4 & 3 & 21,87 \\
Braquignatismo & 5 & 0,0 & 15,6 \\
Prognatismo & 0,0 & 1 & 3,12 \\
Boca de sonrisa & 0,0 & 1 & 3,12 \\
Enojo & 1 & 0,0 & 3,12 \\
Mala erupción & 0,0 & 1 & 3,12 \\
Oligodoncia & 0,0 & 2 & 6,25 \\
Retención de incisivo & 1 & 0,0 & 3,12 \\
Hipoplasia dental (colmillo) & 0,0 & 1 & 3,12 \\
\hline
\end{tabular}

Las espículas dentales u odontofitos, son la patología más frecuente, puesto que se presentó en el $100 \%$ de los equinos estabulados que fueron observados, esta alteración se presenta por el concentrado y granos que generan un desgaste anormal en los premolares y molares formando estas puntas del esmalte dental (Tabla 3).

El cálculo y las rampas se presentaron con mayor frecuencia equinos examinados 68 y $31.25 \%$ respectivamente, siguiendo en frecuencia el sarro $(28 \%)$ que con el cálculo podría verse como una sola patología, debido a que éste tiene su origen en el sarro, y los pacientes que lo tenían fueron los jóvenes. La cuarta patología con mayor frecuencia fueron las ondulaciones $(25 \%)$, luego haba $(21.9 \%)$, las demás enfermedades dentales se presentaron con frecuencias menores al $20 \%$ (Tabla 3). 
Tabla 3. Prevalencia de patologías en la población equina

\begin{tabular}{ccccccc}
\hline \multirow{2}{*}{ Patologías } & \multicolumn{2}{c}{ Yeguas } & \multicolumn{2}{c}{ Caballos } & \multicolumn{2}{c}{$\begin{array}{c}\text { Total población } \\
\text { evaluada }\end{array}$} \\
\cline { 2 - 7 } & No & $\%$ & No & $\%$ & No & $\%$ \\
\hline Sarro dental & 5 & 15.5 & 4 & 12.5 & 9 & 28 \\
Calculo dental & 7 & 21.9 & 15 & 46.9 & 22 & 68,8 \\
Ondulaciones & 3 & 9.4 & 5 & 15.6 & 8 & 25 \\
Rampas & 4 & 12.5 & 6 & 18.75 & 10 & 31,25 \\
Desgaste anormal & 1 & & 5 & & 6 & 18,72 \\
premolares y molares & & 3.12 & & 15.6 & & \\
Gingivitis & 2 & 6.25 & 3 & 9.4 & 5 & 15,65 \\
Diastemas & 1 & 3.12 & 2 & 6.25 & 3 & 9,37 \\
$\begin{array}{c}\text { Incisivos astillados } \\
\begin{array}{c}\text { Fractura de incisivo } \\
\text { Desgaste anormal }\end{array}\end{array}$ & 0,0 & 0,0 & 3 & 9.4 & 3 & 9,4 \\
incisivos & 2 & 6.25 & 2 & 6.25 & 4 & 12,5 \\
Haba & 3 & 9.4 & 4 & 12.5 & 7 & 21,9 \\
$\begin{array}{c}\text { Herida de tejido } \\
\text { blando }\end{array}$ & 0,0 & 0,0 & 3 & 9.4 & 3 & 9,4 \\
\hline
\end{tabular}

Un hallazgo interesante fue incisivos astillados en un macho, posiblemente causado por el estrés de estar encerrado en la pesebrera, generando el desarrollo de algún tipo de "vicio" que terminó ocasionando la patología. Otro caso interesante es la alta prevalencia en los equinos que presentaron haba, puesto que en teoría tienen mayor predisposición a desarrollarla los ejemplares que permanecen en potrero, pero en este estudio el $21.9 \%$ de población afectada por esta patología. Se observaron dos causas frecuentes, la primera es que el equino la desarrolla en potrero antes de llegar a la pesebrera, trasladándolo allí sin hacerle la corrección, y la otra que es más frecuente, la adquieren cuando salen a pastoreo para evitar el estrés, porque los pastos no son adecuados porque generalmente son muy cortos y fibrosos, factores que ocasionan esta patología. 
Es de anotar que ocho caballos presentaban dos patologías en forma simultánea y seis mostraban tres patologías, mientras que en las yeguas fue menor, cuatro y cinco respectivamente (Gráfica 1).

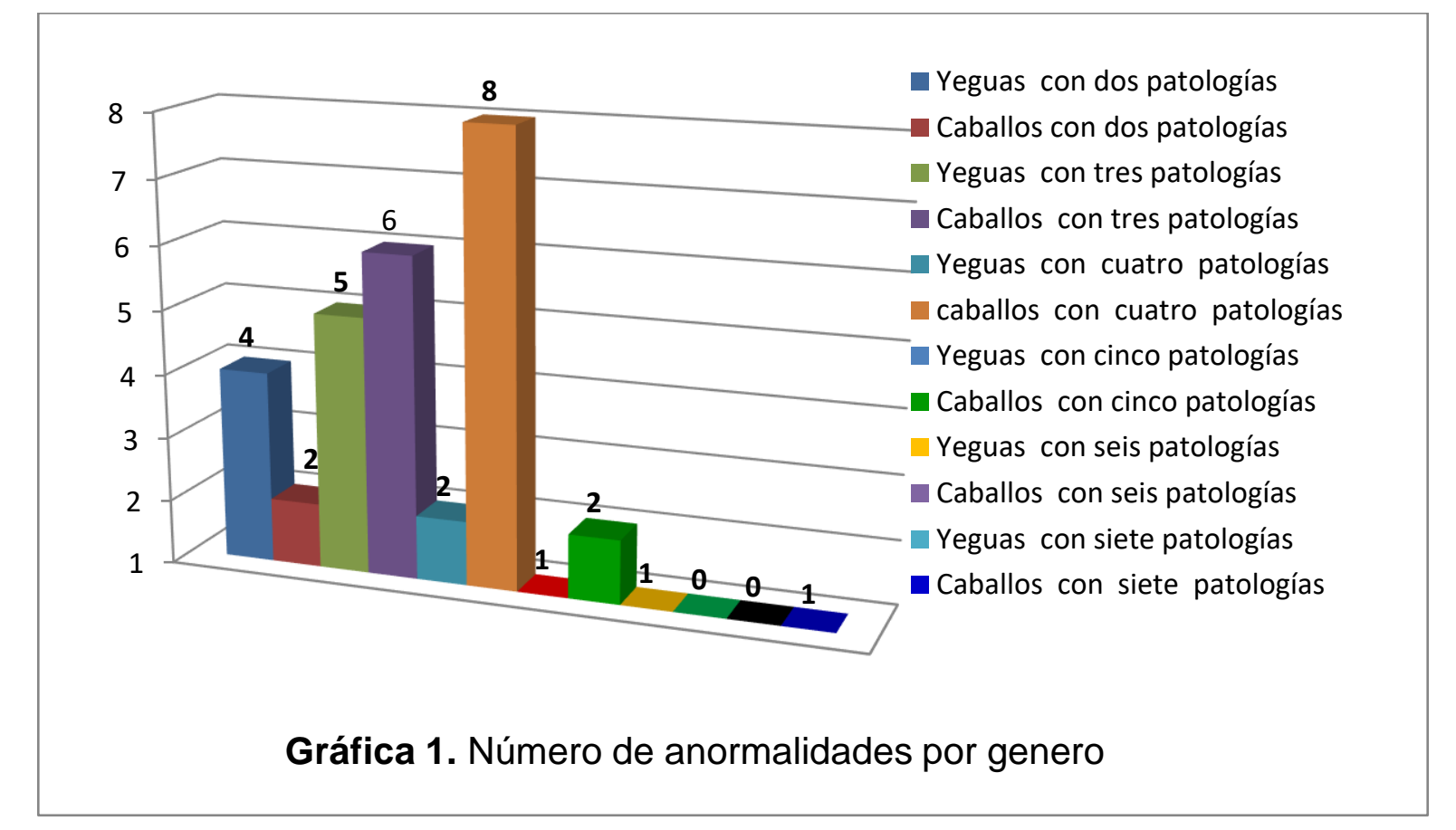

La relación del grupo etario con el número de patología por el sexo y la edad también influyó en la presencia de patologías, es así que se observó en ejemplares machos con más de 9 años de edad, un mayor número de enfermedades odontológicas (Tabla 4 y Gráfica 2)

Tabla 4. Patologías odontológicas por grupo etario

\begin{tabular}{ccccccc}
\hline $\begin{array}{c}\text { Numero } \\
\text { patologías }\end{array}$ & $\begin{array}{c}\text { Yeguas } \\
<\mathbf{5} \text { años }\end{array}$ & $\begin{array}{c}\text { Caballos } \\
<\mathbf{5} \text { años }\end{array}$ & $\begin{array}{c}\text { Yeguas } \\
\mathbf{5} \text { a } \mathbf{9} \\
\text { años }\end{array}$ & $\begin{array}{c}\text { Caballos } \\
\mathbf{5} \text { a } \mathbf{9} \\
\text { años }\end{array}$ & $\begin{array}{c}\text { Yeguas } \\
>\mathbf{9} \text { años }\end{array}$ & $\begin{array}{c}\text { Caballos } \\
>\mathbf{9} \text { años }\end{array}$ \\
\hline 2 & 2 & 1 & 2 & 1 & - & - \\
3 & 2 & 1 & 2 & 3 & - & 2 \\
4 & 1 & 3 & 1 & 2 & 1 & 3 \\
5 & 1 & - & - & 1 & - & 1 \\
6 & - & - & - & - & 1 & - \\
7 & - & - & - & - & - & 1 \\
\hline
\end{tabular}




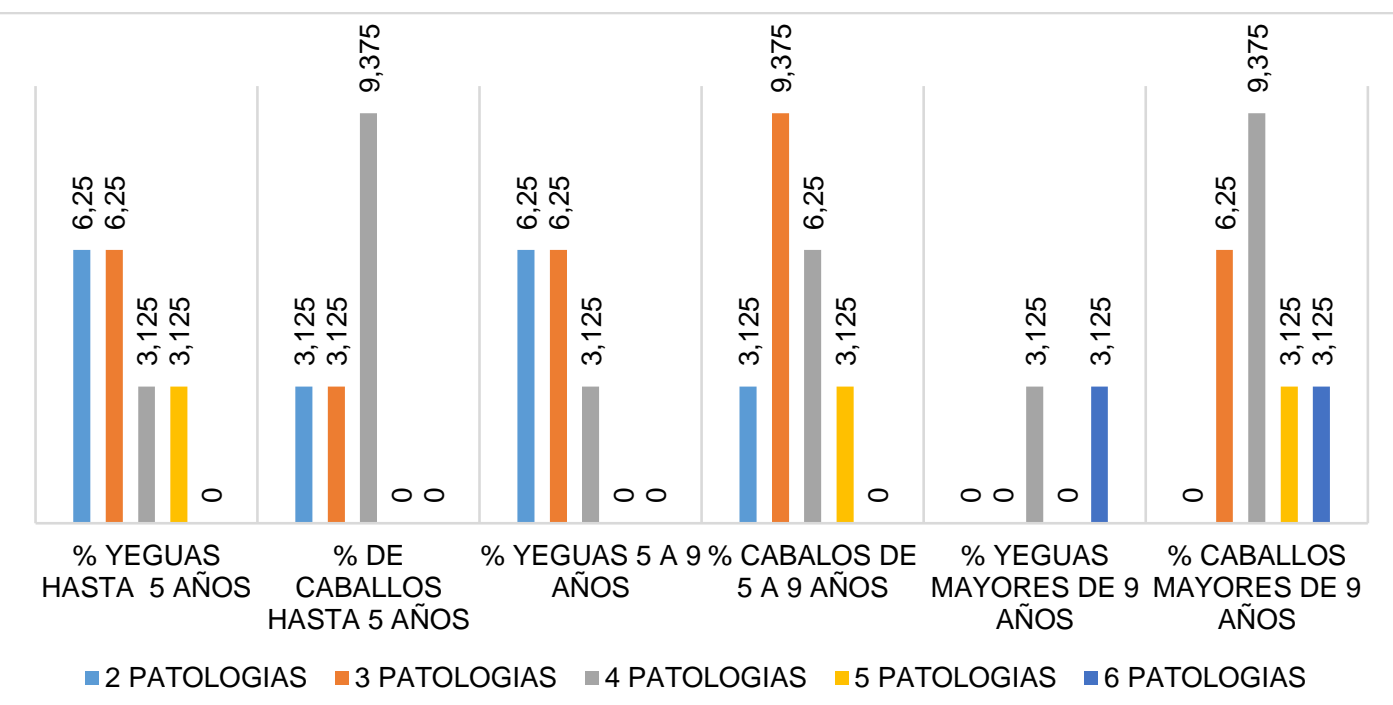

Gráfica 2. Presentación de patologias (\%) por grupo etario

\section{CONCLUSIONES}

El $100 \%$ de los equinos evaluados presentaron alguna patología odontológica, y apenas el $53 \%$ de los propietarios accedió a realizar parte del tratamiento recomendado, destacando el resultado puesto que, mejoraron su peso en $9 \%$ en un periodo de 2 meses, así como su manejo con la rienda, lo que es muy confortable para el propietario.

Hay una relación del género del equino con el braquignatimo, puesto que en este estudio toda la casuística se presentó en yeguas. El sarro y cálculo dental analizados en conjunto son la segunda patología más frecuente en equinos después de las espículas dentales u odontofitos.

Existe una gran relación de las anormalidades y patologías odontológicas con problemas comportamentales y en el manejo de la rienda sin importar cuál sea la actividad que se realice con el equino.

La odontología equina es un campo de acción muy importante para el médico veterinario, puesto que no solo se afecta una parte orgánica del equino, sino que tiene alta incidencia en su comportamiento y en la generación de algunos "vicios" que complica el manejo normal del animal, cuando no se tratan las anormalidades y patologías de los dientes. 


\section{RECOMENDACIONES}

Realizar la profilaxis dental a los equinos estabulados tres veces al año para prevenir problemas tales como el síndrome de abdomen agudo que pueden comprometer la vida de los mismos.

Dentro de la profilaxis dental que se maneja rutinariamente en la profesión, se debe implementar la eliminación del cálculo dental, a pesar de no ser una patología tan grave, si puede conllevar a una enfermedad periodontal.

Realizar una evaluación odontológica a los potros de seis meses de edad con valoración de la mordida para determinar la oclusión y así realizar una corrección a tiempo, lo cual es mejor para el animal y más económico para el propietario.

Es importante disminuir el estrés de los equinos estabulados sacándolos a pastorear o montarlos con mayor frecuencia, porque de lo contrario terminan desarrollando algún "vicio" que afecta principalmente los incisivos.

\section{REFERENCIAS BIBLIOGRÁFICAS}

1. Baker G.J., Easley J. Odontología equina. Editorial Inter-médica, Buenos Aires, Argentina. 2002.

2. Cardona J.A., Álvarez J. Estimación de la edad de los caballos en el examen dentario. Revista UDC A Actualidad \& Divulgación Científica. 13, (1): 29-39. 2010.

3. Collins N., Dixon P. Diagnosis and management of equine diastemata. Clinical techniques in equine practice. 4, (2): 148-154. 2005.

4. Crabill M.R., Schumacher J. Pathophysiology of acquired dental diseases of the horse. The Veterinary clinics of North America. Equine practice. 14, (2): 291-307. 1998.

5. Cruz A.J.M. Enfermedades de los senos paranasales en el caballo. 2004. Recuperado 15 Diciembre $2015 . \quad$ Disponible En: http://albeitar.portalveterinaria.com/noticia/3448/Articulos-otros-temasarchivo/Enfermedades-de-los-senos-paranasales-en-el-caballo.html

6. Cruz A.J.M., Vera L.G., Sánchez J. Enfermedades orales más frecuentes del caballo criollo colombiano. CES Medicina Veterinaria y Zootecnia. 4, (1): 4966. 2010.

7. Dacre I.T. Caries of peripheral cementum. En: Proceedings American Association of Equine Practitioners, p 17-25. 2006.

8. Dixon P. The gross, histological, and ultrastructural anatomy of equine teeth and their relationship to disease. En: Proceedings of the $49^{\text {th }}$ Annual 
Convention of the American Association of Equine Practitioners. 48: 421-437. 2002.

9. Dixon P. The etiology, diagnosis and current therapy of developmental and acquired equine dental disorders. En: $8^{\text {th }}$ Congress on Equine Medicine and Surgery. 2003.

10. Dixon P., Dacre I. A review of equine dental disorders. The veterinary journal. 169, (2): 165-187. 2005.

11. Easley J. Equine canine and first premolar (wolf) teeth. En: $50^{\text {th }}$ Proceeding American Association of Equine Practitioners. 2004.

12. Johnson T.J., Porter C.M. Dental overgrowths and acquired displacement of cheek teeth. En: American Association of Equine Practitioners-Equine Dentistry Focus Meeting, 8. 2006.

13. Klugh D.O. Equine periodontal disease. Clinical techniques in equine practice. 4, (2): 135-147. 2005.

14. Rucker B.A. Treatment of equine diastemata. En: Focus Meeting, 10. 2006. 